\title{
CRÓNICA DEL SEMINARIO DE MEDIACIÓN EN CONFLICTOS LABORALES Y MEDIACIÓN SIMULADA. EVOLUCIÓN DE LA SOLUCIÓN AUTÓNOMA DE CONFLICTOS LABORALES EN LA RIOJA
}

\author{
JORGE RÁBANOS MARTÍNEZ \\ Doctorando, Programa de Doctorado En DERECHO y CAMBio SOCIAL \\ UNIVERSIDAD DE LA RIOJA \\ jorge.rabanos@alum.unirioja.es
}

SUMARIO: I. ABREVIATURAS. II. CRÓNICA DEL SEMINARIO DE MEDIACIÓN EN CONFLICTOS LABORALES Y

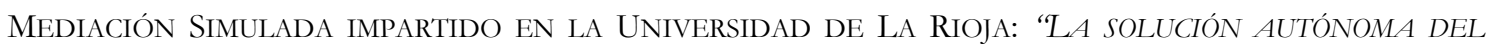
CONFLICTO LABORAL COMO INSTRUMENTO PARA EL TRABAJO DECENTE”. III. SOLUCIÓN AUTÓNOMA DE CONFLICTOS LABORALES EN EL ÁMBITO NACIONAL. IV. SOLUCIÓN AUTÓNOMA DE CONFLICTOS LABORALES EN LA RIOJA. V. ÍNDICE DE RECURSOS UTILIZADOS.

RESUMEN: El presente artículo relata la crónica de las jornadas "La solución autónoma del conflicto laboral como instrumento para el trabajo decente" que se llevaron a cabo en la Universidad de La Rioja en los meses de mayo y junio de 2021. Además, se muestra la evolución de los datos sobre la solución autónoma de conflictos laborales en La Rioja.

PALABRAS CLAVE: Solución autónoma del conflicto laboral, Servicio Interconfederal de Mediación y Arbitraje, Conciliación laboral.

\section{CHRONICLE OF THE SEMINAR ON MEDIATION IN LABOR CONFLICTS AND SIMULATED MEDIATION. EVOLUTION OF THE AUTONOMOUS SETTLEMENT OF LABOR CONFLICTS IN LA RIOJA}

ABSTRACT: This article relates the chronicle of the conference "The autonomous solution of the labor dispute as an instrument for decent work" that took place at the University of La Rioja in the months of May and June 2021. In addition, the evolution is shown of the data on the autonomous resolution of labor disputes in La Rioja.

KEYWORDS: Autonomous solution of the labor dispute, Interconfederal Mediation and Arbitration Service, Work-life balance. 


\section{Abreviaturas}

ASAC: Acuerdo de Solución Autónoma de

Conflictos Laborales.

ASEC: Acuerdo de Solución Extrajudicial de

Conflictos Laborales.

BOE: Boletín Oficial de España.

BOR: Boletín Oficial de La Rioja.

CAR: Comunidad Autónoma de La Rioja.

CCOO: Comisiones Obreras.

CE: Constitución Española.

ET: Estatuto de los Trabajadores.

FSP: Fundación de Servicio Público.

ODS: Objetivos de Desarrollo Sostenible.

ODM: Objetivos de Desarrollo del Milenio.
ONU: Organización de Naciones Unidas.

SIMA: Fundación del Servicio

Interconfederal de Mediación y Arbitraje.

SMAC: Servicio de Mediación, arbitraje y conciliación.

TC: Tribunal Constitucional.

TLR: Tribunal Laboral de La Rioja.

UGT: Unión General de Trabajadores.

UNESCO: Organización de las Naciones

Unidas para la Educación, la Ciencia y la

Cultura.

OIT: Organización Internacional del Trabajo.

\section{Crónica del Seminario de Mediación en Conflictos Laborales y Mediación Simulada impartido en la Universidad de La Rioja: "La solución autónoma del conflicto laboral como instrumento para el trabajo decente"}

A través de la Cátedra UNESCO (Organización de las Naciones Unidas para la Educación, la Ciencia y la Cultura) de la Universidad de La Rioja denominada Cindadanía democrática y libertad cultural se llevó a cabo, bajo la coordinación del Seminario Permanente del Departamento de Derecho, unas jornadas multidisciplinares enmarcadas en el Proyecto de Innovación Docente.

Dichas jornadas estuvieron dirigidas a la formación de los alumnos de cuarto curso del Grado en Relaciones Laborales y Recursos Humanos, siendo extensible a los alumnos del Grado de Derecho interesados en la rama del Derecho del Trabajo, del Master de la Abogacía y del Doctorado en Derecho y Cambio Social.

Se contó con la participación de diferentes organismos como la Fundación SIMA (Servicio Interconfederal de Mediación y Arbitraje), el Tribunal Laboral de La Rioja (TLR), el Ilustre Colegio de Graduados Sociales de La Rioja, la Federación de Empresarios de La Rioja y los dos sindicatos más representativos de La Rioja; CCOO (Comisiones Obreras) y UGT (Unión General de Trabajadores).

Las jornadas se llevaron a cabo los días 18 y 25 de mayo y el 1 de junio de 2021. Su presentación corrió a cargo de la Dra. Dña. M. ${ }^{a}$ Concepción Arruga Segura, coordinadora de las jornadas y Profesora de Derecho del Trabajo y la Seguridad Social de la Universidad de La Rioja. 
El proyecto se centró en el Objetivo de Desarrollo Sostenible (ODS) ${ }^{1}$ número $8^{2}$ de la Agenda 2030 de la Organización de Naciones Unidas (ONU) para promover el trabajo decente.

La iniciativa pretendió impulsar las habilidades negociadoras de los estudiantes de la Universidad de La Rioja ante el conflicto laboral, así como integrar los mecanismos que pueden romper la dinámica de la confrontación y la crispación que invade las relaciones socio laborales.

En el transcurso de la primera jornada, la Dra. Dña. Susana Pérez Escalona, profesora de Derecho Mercantil de la Universidad de La Rioja, defendió su ponencia, titulada La mediación laboral como herramienta de competitividad de las empresas. Una perspectiva desde el Derecho Mercantil. Seguidamente, Dña. Iluminada Ruiz Oca, secretaria técnica del Tribunal Laboral de La Rioja expuso durante la conferencia su punto de vista sobre La aportación del Tribunal Laboral de La Rioja a la solución extrajudicial del conflicto.

Como cierre de la primera sesión del seminario, se produjo un debate en el que los alumnos presentes en las jornadas, tanto de forma presencial como a través del aula virtual de la Universidad, realizaron diversas preguntas sobre la necesidad de fomentar la cultura de la conciliación y la necesidad de dar a conocer al público en general los servicios que ofrece el precitado Tribunal Laboral.

La segunda jornada contó con la presencia de Dña. Beatriz Losada Crespo, directora del Servicio Interconfederal de Mediación y Arbitraje (Fundación SIMA). Durante su exposición, la directora del SIMA profundizó en el papel del Servicio Interconfederal de mediación y arbitraje.

En este sentido, Dña. Beatriz Losada puso de manifiesto la importancia de la reciente firma del VI Acuerdo sobre Solución Autónoma de Conflictos Laborales (ASAC) y, como principal reto, planteó la necesidad de formar a los futuros negociadores de conflictos laborales y responsables de relaciones laborales de empresas y organizaciones en "técnicas de negociación2, "control de los sentimientos" y 2asertividad y empatía para con la otra parte2. En opinión de la ponente, éstos deben ser requisitos mínimos con los que ha de contar un buen negociador en conflictos laborales.

Tras las intervenciones de los ponentes, se llevó a cabo una mesa redonda titulada "El papel de los agentes sociales en la solución autónoma de conflictos2. En ella participaron D. Eduardo Fernández Santolaya, secretario general de la Federación de Empresarios de La Rioja, D. Jesús Izquierdo Santamaría, secretario general de la Unión General de Trabajadores (UGT) en La Rioja y D. Jorge Ruano Chover, secretario general de Comisiones Obreras (CCOO) en La Rioja.

La tercera jornada versó sobre la comunicación como herramienta imprescindible en la solución extrajudicial de conflictos. Para ello, se contó con la presencia de D. Oscar Modrego Pascual, coach motivacional de desarrollo profesional, que llevó a cabo un taller práctico con los alumnos del Grado.

A continuación, y cerrando las jornadas, el presidente del Ilustre Colegio de Graduados Sociales de La Rioja, D. Miguel D’Harcourt, expuso su punto de vista sobre la necesaria implicación del profesional en ejercicio para la solución pacífica de conflictos y comentó algunos casos reales en

\footnotetext{
${ }^{1}$ Los Objetivos de Desarrollo Sostenible (2015-2030), también conocidos por sus siglas ODS, son una iniciativa impulsada por Naciones Unidas para dar continuidad a la agenda de desarrollo tras los Objetivos de Desarrollo del Milenio (ODM). Se pueden consultar los ODS en el siguiente enlace: https://www.un.org/sustainabledevelopment/es/objetivos-de-desarrollo-sostenible/

${ }^{2}$ ODS 8: Trabajo decente y crecimiento económico.
} 
los que tuvo que desarrollarse profesionalmente en las artes de la negociación de conflictos laborales, tanto colectivos como individuales.

El Jornadas Multidisciplinares fueron valoradas de forma muy positiva por los asistentes, por lo novedoso de la temática y por su carácter práctico y cercano que sirvió para dar a conocer la negociación de conflictos entre los ahora estudiantes, pero en un futuro cercano, posibles interlocutores y partes implicadas en dichos procesos de negociación.

\section{Solución Autónoma de Conflictos Laborales en el ámbito nacional}

El Servicio Interconfederal de Mediación y Arbitraje FSP (SIMA-FSP) es, según se desprende de sus propios estatutos, una Fundación estatal que se rige por la Ley 40/2015, de 1 de octubre de Régimen Jurídico del Sector Público y por la Ley 50/2002, de 26 de diciembre, de Fundaciones.

En cumplimiento de lo pactado en el primer Acuerdo sobre Solución Extrajudicial de Conflictos Laborales (ASEC) de 25 de enero de 1996, se constituyó el 14 de julio de 1997 la Fundación Laboral denominada "Fundación del Servicio Interconfederal de Mediación y Arbitraje Fundación de Servicio Público" (SIMA-FSP). Nació como soporte administrativo y de gestión de los procedimientos regulados en el I ASEC, además de ser un espacio de diálogo, negociación y difusión de la cultura de la solución negociada de los conflictos, al amparo de los artículos 7 y 37.2 de la Constitución Española (CE), los Convenios y Recomendaciones de la Organización Internacional del Trabajo (OIT), la Carta Comunitaria de los derechos sociales fundamentales de los trabajadores, el Estatuto de los Trabajadores (ET) y la propia doctrina del Tribunal Constitucional (TC).

Como hito más relevante en el devenir de la Fundación SIMA, cabe reseñar cómo con la aprobación del quinto acuerdo, se incluyó un nuevo enfoque a la solución negociada de conflictos laborales, sacando de la ecuación la visión de que estos acuerdos son la vía extrajudicial previa a la vía judicial, suprimiendo de la nomenclatura de los acuerdos dicho término y pasando a denominarlos ASAC $^{3}$. De esta forma, se destierra la idea de que la judicialización de los conflictos es el cauce final, planteando que la solución puede ser autónoma entre las partes, sin necesidad de intermediación de la Justicia Social.

\section{Solución Autónoma de Conflictos Laborales en el ámbito de La Rioja}

En los artículos 63 y 154 de la Ley 36/2011, de 10 de octubre, reguladora de la jurisdicción social ${ }^{4}$, se delimitan los entes en los que se puede llevar a cabo el intento de conciliación previa

\footnotetext{
3 ASAC: Acuerdo sobre Solución Autónoma de Conflictos laborales por el que se rige la Fundación SIMA. El ASAC es la continuidad de los acuerdos suscritos desde el año 1996, denominados ASEC (Acuerdos de solución extrajudicial de conflictos laborales), por el que se encomienda a la Fundación SIMA la gestión de los procedimientos de mediación y arbitraje.

${ }^{4}$ Artículo 63: Conciliación o mediación previas.

Será requisito previo para la tramitación del proceso el intento de conciliación o, en su caso, de mediación ante el servicio administrativo correspondiente o ante el órgano que asuma estas funciones que podrá constituirse mediante los acuerdos interprofesionales o los convenios colectivos a los que se refiere el artículo 83 del Texto Refundido de la Ley del Estatuto de los Trabajadores, así como mediante los acuerdos de interés profesional a los que se refieren el artículo 13 y el apartado 1 del artículo 18 de la Ley del Estatuto del trabajo autónomo.

Artículo 154. Legitimación activa.
} 
obligatoria. Dicho intento puede realizarse ante el servicio administrativo o ante el órgano que se constituya al efecto mediante acuerdos interprofesionales o por los convenios colectivos a que se refiere el artículo $83^{5}$ del Real Decreto Legislativo 2/2015, de 23 de octubre, por el que se aprueba el texto refundido de la Ley del Estatuto de los Trabajadores.

En La Rioja existen dos entes vinculados a la conciliación-mediación de conflictos laborales; el Servicio de Diálogo Social, Relaciones Laborales y Salud Laboral del Gobierno de La Rioja y el Tribunal Laboral.

El intento de conciliación previa es obligatorio para la tramitación de los procesos derivados de conflictos laborales, individuales y colectivos, ante los órganos jurisdiccionales del orden social, salvo en aquellos exceptuados expresamente por la Ley.

Estas excepciones son:

- Procesos que exijan reclamación administrativa previa

- Procesos en que se demanda al Estado u otro ente público junto a personas privadas, siempre que la pretensión deba someterse a reclamación previa y cuando en la reclamación previa pueda decidirse el asunto litigioso

- En materias de Seguridad Social.

- Disfrute de vacaciones

- Materia electoral

- Los iniciados de oficio

- Impugnación de convenios colectivos

- Impugnación de estatutos de los sindicatos o de su modificación

- Tutela de la libertad sindical

Estarán legitimados para promover procesos sobre conflictos colectivos:

a) Los sindicatos cuyo ámbito de actuación se corresponda o sea más amplio que el del conflicto.

b) Las asociaciones empresariales cuyo ámbito de actuación se corresponda o sea más amplio que el del conflicto, siempre que se trate de conflictos de ámbito superior a la empresa.

c) Los empresarios y los órganos de representación legal o sindical de los trabajadores, cuando se trate de conflictos de empresa o de ámbito inferior.

d) Las Administraciones públicas empleadoras incluidas en el ámbito del conflicto y los órganos de representación del personal laboral al servicio de las anteriores.

e) Las asociaciones representativas de los trabajadores autónomos económicamente dependientes y los sindicatos representativos de estos, para el ejercicio de las acciones colectivas relativas a su régimen profesional, siempre que reúnan el requisito de la letra a) anterior, así como las empresas para las que ejecuten su actividad y las asociaciones empresariales de éstas siempre que su ámbito de actuación sea al menos igual al del conflicto.

${ }^{5}$ Artículo 83. Unidades de negociación.

2. Las organizaciones sindicales y asociaciones empresariales más representativas, de carácter estatal o de comunidad autónoma, podrán establecer, mediante acuerdos interprofesionales, cláusulas sobre la estructura de la negociación colectiva, fijando, en su caso, las reglas que han de resolver los conflictos de concurrencia entre convenios de distinto ámbito.

Estas cláusulas podrán igualmente pactarse en convenios o acuerdos colectivos sectoriales, de ámbito estatal o autonómico, por aquellos sindicatos y asociaciones empresariales que cuenten con la legitimación necesaria, de conformidad con lo establecido en esta ley. 
- Procesos ya iniciados, en los que hubiera que dirigir la demanda frente a personas distintas de las inicialmente demandadas

El trabajador dispondrá de 20 días hábiles para solicitar la convocatoria del acto de conciliación. El plazo empezará a contar a partir del día en que las acciones pudieran ejercitarse y que, en principio, es el del hecho determinante de la reclamación, para los casos de despido, sanciones impuestas por el empresario en el ejercicio de su facultad disciplinaria y decisiones empresariales en materia de movilidad geográfica y modificación de condiciones sustanciales de trabajo.

En el caso de reclamación de cantidades y otras acciones derivadas del contrato de trabajo que no tengan señalado plazo especial, el trabajador dispondrá de un año de plazo para cursar la solicitud de acto de conciliación.

La asistencia al acto de conciliación es obligatoria para ambas partes y la no comparecencia del solicitante da lugar al archivo de lo actuado, teniéndose por no presentada la papeleta. La incomparecencia de la otra parte supone que la conciliación ha sido "intentada sin efecto" y queda vía libre al solicitante para presentar la demanda.

Lo acordado en conciliación, puede ser impugnado, tanto por cualquiera de las partes, como por terceros que pudieran sufrir algún perjuicio, ante el Juzgado de lo Social.

$\mathrm{El}$ acuerdo entre las partes intervinientes tiene fuerza ejecutiva sin que tenga que ratificarse ante el órgano judicial.

A continuación, se realizará una breve aproximación a estos dos entes y a los informes de evolución que anualmente emiten:

\section{IV.1. Servicio de Diálogo Social, Relaciones Laborales y Salud Laboral del Gobierno de La Rioja}

El de mayor antigüedad, heredero del Servicio de Mediación, Arbitraje y Conciliación del Gobierno de La Rioja, es el Servicio de Diálogo Social, Relaciones Laborales y Salud Laboral de la Consejería de Desarrollo Autonómico del Gobierno de La Rioja, que nace al amparo del Decreto 46/2020, de 3 de septiembre, por el que se establece la estructura orgánica de la Consejería de Desarrollo Autonómico y sus funciones en desarrollo de la Ley 3/2003, de 3 de marzo, de Organización del Sector Público de la Comunidad Autónoma de La Rioja (publicado en el BOR de 4 de septiembre de 2020).

El Servicio de Diálogo Social, Relaciones Laborales y Salud Laboral, se presta bajo la Dirección General de Empleo, Diálogo Social y Relaciones Laborales de la Consejería de Desarrollo Autonómico del Gobierno de la Comunidad Autónoma de La Rioja.

La legislación específica por la que se rige el Servicio es la Ley 36/2011, de 10 de octubre, reguladora de la jurisdicción social (en vigor desde el 11 de diciembre de 2011) y el Real Decreto 2756/1979, de 23 de noviembre, sobre asunción de funciones por el Instituto de Mediación, Arbitraje y Conciliación.

El Servicio de Diálogo Social, Relaciones Laborales y Salud Laboral del Gobierno de La Rioja tiene como propósito el intento previo ante órganos administrativos, de llegar a un acuerdo satisfactorio entre los trabajadores y las empresas con el fin de evitar la intervención judicial. 
El acto de conciliación se realiza ante un letrado-conciliador, mediante la presentación de la demanda en el registro, el que en ese instante hace entrega de la copia sellada con expresión del día y la hora en la que se celebrará el acto y remite la correspondiente citación a la parte interesada.

Alcanzada la conciliación, lo acordado en ella constituye título suficiente para ejecutarse directamente en los mismos términos que se ejecutan las sentencias.

\section{IV.1.1. Informe anual del Servicio de Diálogo Social, Relaciones Laborales y Salud Laboral del año 2020}

Anualmente, el Servicio emite un informe ${ }^{6}$ en el que se muestran datos de evolución sobre la conciliación administrativa previa a la vía judicial, diferenciando los datos entre "Conciliaciones en conflictos individuales 2 y "Conciliaciones en conflictos colectivos".

El último informe disponible, al que se puede acceder bajo petición formal al Servicio, recoge datos una comparativa de datos entre los años 2016 y 2020, estudiando así la tendencia y evolución durante un espectro temporal de cinco años.

\section{IV.1.2. Datos sobre actividad del Servicio de Diálogo Social, Relaciones Laborales y Salud Laboral}

\section{a. Conciliaciones en conflictos individuales}

La conciliación por conflictos individuales registrada por el Gobierno de La Rioja a lo largo del año 2020 sufrió un descenso relevante, como consecuencia de la declaración el Estado de Alarma ${ }^{7}$ por el Gobierno de España en el mes de marzo (bajada significativa en los meses de marzo y abril) y la consiguiente reducción de trámites administrativos.

La evolución de los últimos años con respecto al año 2020 estaría equilibrada y acompasada con la tendencia de los años inmediatamente anteriores, pero como consecuencia de la pandemia de la COVID-19 se percibe un descenso relevante en los datos de 2020 con respecto a los datos de los años anteriores.

\footnotetext{
6 Informe de acceso condicionado a la previa solicitud formal del mismo al Servicio de Diálogo Social, Relaciones Laborales y Salud Laboral del Gobierno de La Rioja. No se encuentra publicado en la página web del Gobierno de La Rioja, por lo que su acceso por parte de la ciudadanía no es directo a través de Internet.

${ }^{7}$ En base al Real Decreto 463/2020, de 14 de marzo, por el que se declara el estado de alarma para la gestión de la situación de crisis sanitaria ocasionada por el Covid-19.
} 
Tabla 1. CAR - Evolución mensual de Conciliaciones en conflictos individuales.

\begin{tabular}{|l|c|c|c|c|c|c|c|c|c|}
\hline & $\mathbf{2 0 1 6}$ & $\mathbf{2 0 1 7}$ & $\begin{array}{c}\mathbf{\%} \\
\mathbf{2 0 1 6 / 2 0 1 7}\end{array}$ & $\mathbf{2 0 1 8}$ & $\begin{array}{c}\mathbf{\%} \\
\mathbf{2 0 1 7 / 2 0 1 8}\end{array}$ & $\mathbf{2 0 1 9}$ & $\begin{array}{c}\mathbf{\%} \\
\mathbf{2 0 1 8 / 2 0 1 9}\end{array}$ & $\mathbf{2 0 2 0}$ & $\begin{array}{c}\mathbf{\%} \\
\mathbf{2 0 1 9 / 2 0 2 0}\end{array}$ \\
\hline Enero & 158 & 150 & $-5,06 \%$ & 177 & $18,00 \%$ & 133 & $-24,85 \%$ & 155 & $16,45 \%$ \\
\hline Febrero & 197 & 151 & $-23,35 \%$ & 160 & $5,96 \%$ & 228 & $42,50 \%$ & 145 & $-36,40 \%$ \\
\hline Marzo & 129 & 134 & $3,88 \%$ & 139 & $3,73 \%$ & 131 & $-5,76 \%$ & 89 & $-32,61 \%$ \\
\hline Abril & 166 & 91 & $-45,18 \%$ & 133 & $46,15 \%$ & 171 & $28,57 \%$ & 0 & $-100 \%$ \\
\hline Mayo & 241 & 150 & $-37,76 \%$ & 154 & $2,66 \%$ & 158 & $2,60 \%$ & 0 & $-100 \%$ \\
\hline Junio & 109 & 128 & $17,43 \%$ & 144 & $12,50 \%$ & 133 & $-7,63 \%$ & 220 & $65,41 \%$ \\
\hline Julio & 176 & 234 & $32,95 \%$ & 123 & $-47,44 \%$ & 201 & $63,41 \%$ & 203 & $0,99 \%$ \\
\hline Agosto & 182 & 150 & $-17,58 \%$ & 156 & $4,00 \%$ & 135 & $-13,46 \%$ & 84 & $-37,78 \%$ \\
\hline Septiembre & 166 & 112 & $-32,53 \%$ & 110 & $-1,79 \%$ & 111 & $0,91 \%$ & 119 & $7,20 \%$ \\
\hline Octubre & 147 & 155 & $5,44 \%$ & 135 & $-12,90 \%$ & 143 & $5,92 \%$ & 141 & $-1,10 \%$ \\
\hline Noviembre & 161 & 173 & $7,45 \%$ & 136 & $-21,39 \%$ & 114 & $-16,18 \%$ & 110 & $-3,50 \%$ \\
\hline Diciembre & 97 & 134 & $38,14 \%$ & 125 & $-6,72 \%$ & 110 & $-12,00 \%$ & 125 & $13,64 \%$ \\
\hline \multicolumn{1}{|c|}{ TOTAL } & $\mathbf{1 . 9 2 9}$ & $\mathbf{1 . 7 6 2}$ & $\mathbf{- 8 , 6 0 \%}$ & $\mathbf{1 . 6 9 2}$ & $\mathbf{- 3 , 9 7 \%}$ & $\mathbf{1 . 7 6 8}$ & $\mathbf{4 , 4 9 , \%}$ & $\mathbf{1 3 9 1}$ & $\mathbf{- 2 0 7 , 7 0 \%}$ \\
\hline
\end{tabular}

Se pueden observar las conciliaciones presentadas durante los años 2016, 2017, 2018, 2019 y 2020, incluyéndose las presentadas por la Fundación Laboral de la Construcción ${ }^{8}$ sobre reclamaciones a los empresarios del pago de las cuotas establecidas en los convenios del sector de la construcción; 99 en el ejercicio 2016, 42 en el ejercicio 2017, 6 en el ejercicio 2018, 90 en 2019 y ninguna en 2020.

Descontando los citados actos, el número de conciliaciones celebradas con trabajadores y su impacto porcentual con respecto a los datos del año anterior fueron los siguientes:

Tabla 2. CAR - Evolución anual de Conciliaciones en conflictos individuales.

\begin{tabular}{|l|c|c|c|c|c|}
\cline { 2 - 6 } \multicolumn{1}{c|}{} & \multicolumn{5}{c|}{ AÑ } \\
\cline { 2 - 6 } \multicolumn{1}{c|}{} & $\mathbf{2 0 1 6}$ & $\mathbf{2 0 1 7}$ & $\mathbf{2 0 1 8}$ & $\mathbf{2 0 1 9}$ & $\mathbf{2 0 2 0}$ \\
\hline $\mathrm{N}^{\mathrm{o}}$ expedientes tramitados & 1.830 & 1.720 & 1.686 & 1.678 & 1.391 \\
\hline Porcentaje con respecto al año anterior & - & $-5,60 \%$ & $-1,98 \%$ & $-0,47 \%$ & - \\
\hline
\end{tabular}

En el año 2020, mayoritariamente (873 de un total de 1.435), la temática que generó más conflictos individuales fueron los "despidos", seguida de la reclamación de "cantidades" (444 de $1.435)^{9}$.

\footnotetext{
8 Unión de la Confederación Nacional de la Construcción (CNC), CCOO del Hábitat y la Federación de Industria, Construcción y Agro de UGT (UGT FICA), que da forma a esta entidad paritaria sin ánimo de lucro. Más información en: https://www.fundacionlaboral.org/

${ }^{9}$ Los datos relativos a un año en concreto, excluyen del cómputo los expedientes sin tramitar en dicho año, pero con origen en el año anterior. Del mismo modo, se excluyen aquellos expedientes que, habiendo tenido origen en el año en curso, no se han podido tramitar y han quedado pendientes de resolución para el ejercicio siguiente.
} 
Tabla 3. CAR - Conflictos individuales gestionados.

\begin{tabular}{|l|c|c|c|c|c|}
\cline { 3 - 5 } \multicolumn{1}{c|}{} & \multicolumn{3}{c|}{$\begin{array}{c}\text { CAUSAS DE LOS CONFLICTOS } \\
\text { INDIVIDUALES }\end{array}$} \\
\cline { 2 - 5 } \multicolumn{1}{c|}{} & $\begin{array}{c}\text { PENDIENTES } \\
\text { AÑO ANTERIOR }\end{array}$ & $\begin{array}{c}\text { PRESENTADOS } \\
\text { EN EL AÑO }\end{array}$ & SUMA & $\begin{array}{c}\text { ASUNTOS } \\
\text { TERMINADOS }\end{array}$ & $\begin{array}{c}\text { PENDIENTES } \\
\text { ANNO SIGUIENTE }\end{array}$ \\
\hline Despidos & 31 & 842 & 873 & 850 & 23 \\
\hline Sanciones & 1 & 51 & 52 & 52 & 0 \\
\hline Cantidades & 26 & 418 & 444 & 424 & 20 \\
\hline Varios & 0 & 66 & 66 & 65 & 1 \\
\hline TOTALES & $\mathbf{5 8}$ & $\mathbf{1 3 7 7}$ & $\mathbf{1 4 3 5}$ & $\mathbf{1 3 9 1}$ & $\mathbf{4 4}$ \\
\hline
\end{tabular}

El resultado de todos estos procesos de conciliación administrativa previa a la vía judicial durante el año 2020 fue el siguiente; Con avenencia ${ }^{10}, 616$ expedientes. Sin avenencia, 436 expedientes. Intentados sin efecto, 263 expedientes. Otros motivos, 76 expedientes. En la siguiente table puede observarse dicha situación:

Tabla 4. CAR - Resultado de los procesos de Conciliación por conflictos individuales.

\begin{tabular}{|c|r|r|r|r|r|}
\hline \multicolumn{7}{|c|}{ RES ULTADOS CONCILIACIONES REALIZADAS POR CONFLICTOS INDIVIDUALES } \\
\hline \multirow{2}{*}{ RESULTADO } & \multicolumn{3}{|c|}{ MOTIVO } & VARIOS & TOTALES \\
\cline { 2 - 7 } & DESPIDOS & SANCIONES & CANTIDADES & & 1 \\
\hline Con avenencia & 553 & 9 & 53 & 48 & $\mathbf{6 1 6}$ \\
\hline Sin avenencia & 172 & 35 & 181 & 12 & $\mathbf{2 6 3}$ \\
\hline $\begin{array}{c}\text { Intentado sin } \\
\text { efecto }\end{array}$ & 100 & 6 & 145 & 4 & $\mathbf{7 6}$ \\
\hline Otros motivos & 25 & 2 & 45 & $\mathbf{6 5}$ & $\mathbf{1 . 3 9 1}$ \\
\hline S UMAN & $\mathbf{8 5 0}$ & $\mathbf{5 2}$ & $\mathbf{4 2 4}$ & & \\
\hline
\end{tabular}

En la siguiente tabla, puede observarse la evolución desde el año 2016 según el resultado obtenido en las diferentes causas que motivan la conciliación:

\footnotetext{
${ }^{10}$ Según la Nota Explicativa emitida la Subdirección General de Estadística y Análisis Sociolaboral de la Secretaría de Estado de Empleo y Economía Social del Ministerio de Trabajo y Economía Social en lo referente a Mediación, Arbitraje y Conciliación (MAC), define los siguientes conceptos tal que así: Con avenencia, cuando se dé acuerdo entre las partes. Sin avenencia, cuando no exista acuerdo entre las partes. Intentadas sin efecto, cuando no comparezca la parte o partes demandadas. Tenidas por no presentadas, cuando, debidamente citadas las partes, la promotora no comparezca al acto. Desistidas, cuando comparezca la parte promotora al sólo efecto de desistir del conflicto planteado. La precitada nota explicativa puede ser consultada en: https://expinterweb.mitramiss.gob.es/series/pdf/33-FyN.pdf
} 
Tabla 5. CAR - Evolución de resoluciones de conflictos individuales por causa.

\begin{tabular}{|c|r|r|r|r|c|}
\hline AÑO & \multicolumn{1}{|c|}{$\mathbf{2 0 1 6}$} & $\mathbf{2 0 1 7}$ & \multicolumn{2}{|c|}{$\mathbf{2 0 1 8}$} & \multicolumn{2}{|c|}{$\mathbf{2 0 1 9}$} & $\mathbf{2 0 2 0}$ \\
\hline Con avenencia & 632 & 663 & 709 & 747 & 553 \\
\hline Sin avenencia & 192 & 194 & 164 & 520 & 172 \\
\hline Intentado sin efecto & 114 & 95 & 83 & 405 & 100 \\
\hline Tenidas por no presentadas & \multicolumn{1}{|c|}{-} & - & - & 96 & - \\
\hline Desistidas & - & - & - & - & - \\
\hline Otros motivos & 19 & 22 & 18 & - & 25 \\
\hline TOTAL & 957 & 974 & 974 & 1.768 & 850 \\
\hline
\end{tabular}

El resumen de conflictos individuales según la causa que los generó, desde el año 2016 al año 2020, fue el siguiente:

Tabla 6. CAR - Conflictos individuales según la causa que los generó.

\begin{tabular}{|l|c|c|c|c|c|}
\hline \multicolumn{7}{|c|}{ CAUSAS CONFLICTOS INDIVIDUALES } \\
\hline & $\mathbf{2 0 1 6}$ & $\mathbf{2 0 1 7}$ & $\mathbf{2 0 1 8}$ & $\mathbf{2 0 1 9}$ & $\mathbf{2 0 2 0}$ \\
\hline Despidos & 957 & 974 & 974 & 1.014 & 850 \\
\hline Sanciones & 57 & 56 & 61 & 45 & 52 \\
\hline Cantidades & 767 & 637 & 535 & 597 & 424 \\
\hline Varios & 170 & 95 & 122 & 112 & 65 \\
\hline Total & $\mathbf{1 . 9 5 1}$ & $\mathbf{1 . 7 6 2}$ & $\mathbf{1 . 6 9 2}$ & $\mathbf{1 . 7 6 8}$ & $\mathbf{1 . 3 9 1}$ \\
\hline
\end{tabular}

El resultado de las conciliaciones individuales desde el año 2016 hasta el año $2019^{11}$ se mantuvo en equilibrio, siendo en 2020 cuando se produjo un descenso significativo.

Cabe reseñar las características de la zona geográfica a la que hacen referencia los datos, pudiéndose dar en la Comunidad Autónoma de La Rioja diferencias numéricas y porcentuales significativas como consecuencia de un número reducido de expedientes.

Tabla 7. CAR - Resultado de las conciliaciones individuales.

\begin{tabular}{|c|c|c|c|c|c|}
\cline { 2 - 6 } \multicolumn{1}{c|}{} & \multicolumn{5}{c|}{ RESULTADOS CONCILIA CIONES INDIVIDUALES } \\
\cline { 2 - 6 } \multicolumn{1}{c|}{} & $\mathbf{2 0 1 6}$ & $\mathbf{2 0 1 7}$ & $\mathbf{2 0 1 8}$ & $\mathbf{2 0 1 9}$ & $\mathbf{2 0 2 0}$ \\
\hline CON A VENENCIA & 780 & 778 & 810 & 747 & 616 \\
\hline SIN A VENENCIA & 485 & 528 & 480 & 520 & 436 \\
\hline INTENTADO SIN EFECTO & 583 & 377 & 327 & 405 & 263 \\
\hline TENIDA S POR NO PRESENT. & & & & 0 & 0 \\
\hline DESISTIDAS & & & & 0 & 0 \\
\hline OTROS MOTIVOS & 103 & 79 & 75 & 96 & 76 \\
\hline TOTAL & $\mathbf{1 . 9 5 1}$ & $\mathbf{1 . 7 6 2}$ & $\mathbf{1 6 9 2}$ & $\mathbf{1 . 7 6 8}$ & $\mathbf{1 . 3 9 1}$ \\
\hline
\end{tabular}

\footnotetext{
${ }^{11}$ Teniendo en cuenta la matización realizada en la nota al pie número 8.
} 


\section{a. Conciliaciones en conflictos colectivos}

Durante el año $2020^{12}$, se han realizado las actuaciones sobre conflictos colectivos que a continuación se detallan:

Tabla 8. CAR. Conflictos colectivos gestionados.

\begin{tabular}{|c|c|c|}
\cline { 2 - 3 } \multicolumn{1}{c|}{} & CONFLICTOS & TRABAJADORES AFECTADOS \\
\hline Pendientes año anterior & 1 & 130 \\
\hline Presentados en el año 2020 & 11 & 1894 \\
\hline Pendientes año siguiente & 1 & 126 \\
\hline TOTAL & $\mathbf{1 3}$ & $\mathbf{2 . 1 5 0}$ \\
\hline
\end{tabular}

El resultado de estos procesos de negociación de conflictos colectivos ha sido, en su mayoría, sin avenencia, quedando latente de esta forma la complejidad de este tipo de conflictos en comparación con los resultados obtenidos cuando el conflicto en cuestión afecta al individuo:

Tabla 9. CAR - Resultado de las conciliaciones colectivas.

\begin{tabular}{|c|c|c|c|c|c|}
\hline & \multicolumn{5}{|c|}{ RESULTADOS ANUALES DE CONCILIACIONES COLECTIVAS } \\
\hline & 2016 & 2017 & 2018 & 2019 & 2020 \\
\hline Con avenencia & & 1 & & & \\
\hline Sin avenencia & 7 & 2 & 3 & 3 & 10 \\
\hline Intentado sin efecto & 1 & & 2 & & \\
\hline $\begin{array}{l}\text { Tenidas por no } \\
\text { presentadas }\end{array}$ & 0 & 0 & 0 & 0 & 0 \\
\hline Desistidas & 0 & 0 & 0 & 0 & 1 \\
\hline Otros motivos & 0 & 0 & 0 & 0 & \\
\hline TOTAL & 8 & 3 & 5 & 3 & 11 \\
\hline
\end{tabular}

Se puede apreciar que la tendencia en el caso de los conflictos colectivos ha aumentado de manera exponencial en el año 2020 como consecuencia de la pandemia por Covid-19 y los ERTES ${ }^{13}$ que ésta generó y que fueron regulados mediante el Real Decreto-Ley 8/2020, de 17 de marzo, en el que se definieron las condiciones específicas para los Expedientes de Regulación Temporal de Empleo.

\footnotetext{
12 Teniendo en cuenta la matización realizada en la nota al pie número 8.

13 El Expediente de Regulación Temporal de Empleo, más conocido como ERTE, es una medida de flexibilización laboral que habilita a la empresa para reducir o suspender los contratos de trabajo. Este procedimiento queda circunscrito a un periodo de tiempo debidamente acotado, a la conclusión del cual la empresa está obligada a recuperar las condiciones contractuales previas a la implementación del ERTE, así como a mantener los puestos de trabajo de los empleados que se han visto afectados. Durante la pandemia por la Covid-19 este recurso de gestión de crisis en el ámbito laboral fue muy popular, por el alto número de afectados a los que se les aplicó.
} 
El número de empresas y de trabajadores afectados por los conflictos colectivos que se produjeron durante el año 2020 fue muy superior al que se venía dando en los años anteriores; Concretamente, 1.898 trabajadores afectados frente a los 352 afectados en el año 2016.

Tabla 10. CAR - Resultado de las conciliaciones en conflictos colectivos según número de empresas y número de trabajadores afectados.

\begin{tabular}{|c|c|c|c|c|c|c|}
\hline & \multirow{2}{*}{ AÑ̃ } & \multirow{2}{*}{$\mathbf{N}^{\mathbf{0}}$} & \multirow{2}{*}{$\mathbf{N}^{\mathbf{0}}$} & \multicolumn{4}{|c|}{ RESULTADO } \\
\cline { 5 - 7 } & EMPRESAS & $\begin{array}{c}\text { TRABAJADORES } \\
\text { AFECTADOS }\end{array}$ & $\begin{array}{c}\text { CON } \\
\text { AVENENCIA }\end{array}$ & $\begin{array}{c}\text { SIN } \\
\text { AVENENCIA }\end{array}$ & $\begin{array}{c}\text { INTENTADO } \\
\text { SIN EFECTO }\end{array}$ & $\begin{array}{c}\text { OTROS } \\
\text { MOTIVOS }\end{array}$ \\
\hline 2016 & 8 & 352 & & 7 & 1 & \\
\hline 2017 & 3 & 358 & 1 & 2 & & \\
\hline 2018 & 5 & 875 & & 3 & 2 & \\
\hline 2019 & 3 & 349 & & 3 & & \\
\hline 2020 & 11 & 1898 & & 10 & & 1 \\
\hline
\end{tabular}

\section{IV.2. Tribunal Laboral de La Rioja}

Por Acuerdo Interprofesional de 23 de noviembre de $1994^{14}$ suscrito por la Federación de Empresarios de La Rioja y los Sindicatos Unión General de Trabajadores de La Rioja y Unión Regional de Comisiones Obreras de La Rioja, se acordó la constitución del Tribunal Laboral de Conciliación, Mediación y Arbitraje ${ }^{15}$ de La Rioja (en adelante TLR) como órgano de solución extrajudicial y extraadministrativo de conflictos laborales.

La composición, funciones y procedimientos de actuación del TLR se regulan en base al reglamento ${ }^{16}$ publicado en el Boletín Oficial de La Rioja de fecha 2 de enero de 1997.

El TLR es un órgano extrajudicial de solución de conflictos laborales, mediante la mediación y el arbitraje al amparo del artículo 63 de la Ley 36/2011, 10 de octubre, reguladora de la Jurisdicción Social.

Se sustenta por medio de una Fundación privada ${ }^{17}$ constituida por los firmantes del Acuerdo Interprofesional y bajo la tutela del Gobierno de La Rioja.

\footnotetext{
${ }^{14}$ Boletín Oficial de la Rioja número 160, página 4.780, de 31 de diciembre de 1994. Puede consultarse el boletín en: http://ias1.larioja.org/boletin/Bor_Boletin_visor_Servlet?referencia=515017-1-PDF-76412

15 Aunque así es como se denomina, en la actualidad no existe la posibilidad de arbitraje en el TLR.

${ }^{16}$ Los acuerdos del Comité Paritario de Interpretación, Aplicación y Seguimiento del Acuerdo Interprofesional de La Rioja de fecha 15 de mayo de 1997 conllevaron modificaciones del reglamento primigenio. Dichos acuerdos fueron publicados en el Boletín Oficial de La Rioja número 94, de 7 de agosto de 1997.

${ }^{17}$ Así consta en el Registro de Fundaciones del Gobierno de La Rioja. En dicho registro se define la siguiente finalidad del TLR: "Facilitar los medios necesarios para que el Tribunal Laboral pueda ejercer las funciones de mediación, conciliación y arbitraje a beneficio de las empresas y trabajadores de La Rioja, tanto en los conflictos colectivos como en los individuales, que se produzcan en el ámbito de las relaciones laborales de La Rioja". En lo relativo a sus funciones, en el registro constan las propias de "mediación, conciliación y arbitraje a beneficio de las empresas y trabajadores de La Rioja, tanto en los conflictos colectivos como en los individuales, que se produzcan en el ámbito de las relaciones laborales de La Rioja". El precitado registro puede consultarse en: https://www.larioja.org/es/integra2?dato $=854$
} 
La financiación del TLR está recogida anualmente en los presupuestos Generales de la Comunidad Autónoma de La Rioja.

El TLR es un organismo autónomo ${ }^{18}$ encargado de gestionar las solicitudes que las partes presentan para llevar a cabo procesos de negociación de conflictos, a fin de evitar la resolución de los mismos por cauces judiciales. El cometido esencial del TLR, como así se detalla en sus Estatutos, es el de "promover, facilitar y proporcionar los sistemas, mecanismos y prácticas de solución voluntarias, de los conflictos laborales, individuales o colectivos, dando trámite así a la preceptiva conciliaciónmediación previa a la vía judicial". Se rige por los principios de voluntariedad, gratuidad, igualdad, imparcialidad, autonomía, inmediatez, audiencia y contradicción.

Los cauces en base a los que lleva a cabo su actividad el TLR son:

1. Conciliación-mediación:

En el caso de la conciliación-mediación, la parte interesada en iniciar el proceso deberá cursar la solicitud por escrito, haciendo constar los datos identificativos de las partes en cuestión, y un texto introductorio con el que se pueda apreciar la naturaleza del conflicto y su magnitud. El TLR citará a las partes para la celebración del acto de conciliaciónmediación.

2. Arbitraje ${ }^{19}$ :

En el caso del arbitraje, se puede acceder a este método de búsqueda extrajudicial de conflictos colectivos mediante dos vías.

- Directamente, mediante la presentación voluntaria de un escrito firmado por ambas partes en conflicto en el que acaten y se obliguen a cumplir la decisión final emitida por el árbitro.

- La otra forma de acceder a este método es una vez agotado el trámite de la Mediación-Conciliación sin llegar a acuerdo. En este caso también se exigen los dos requisitos de sometimiento expreso y obligación de acatamiento. El laudo tendrá carácter vinculante y fuerza ejecutiva.

El TLR tiene tres objetivos:

1. Mediación, Conciliación y Arbitraje, tanto en los conflictos colectivos como individuales que se produzcan en el ámbito de las relaciones laborales.

2. Divulgación y fomento de los procesos de solución extrajudicial de conflictos en el ámbito de las relaciones laborales.

3. Facilitación de la negociación colectiva y actuaciones de las Comisiones Paritarias, así como la resolución de conflictos laborales.

\footnotetext{
${ }^{18}$ Desde el punto de vista de la gestión, ya que económicamente depende de la financiación autonómica que se delimite en los Presupuestos Generales de La Rioja. Dicha financiación se lleva a cabo mediante la firma de un Convenio de Colaboración entre el Gobierno de La Rioja y el propio Tribunal Laboral de Conciliación, Mediación y Arbitraje de La Rioja.

${ }^{19}$ Teniendo en cuenta la matización realizada en la nota al pie número 14.
} 
Pueden acogerse a los servicios prestados por el TLR los trabajadores y empresarios de forma individual, o bien a través de sus órganos de representación o sus organizaciones representativas y podrán solicitar la conciliación-mediación o el arbitraje.

\section{IV.2.1. Informe anual del Tribunal Laboral de La Rioja para el año 2020}

En el apartado "Transparencia” de la página web del TLR se recogen las Cuentas Anuales del año 2019, el Plan de Actuación del año 2020 y el Balance del Tribunal Laboral de los años 2019 y 2020.

En el precitado Balance ${ }^{20}$ del año 2020 pueden observarse datos referentes a los expedientes gestionados durante el ejercicio, pudiéndose extraer relevantes conclusiones:

\section{IV.2.2. Datos sobre actividad del Tribunal Laboral de La Rioja}

Pese a la paralización global sufrida como consecuencia del Estado de Alarma, la actividad del TLR se vio incrementada en un 1,93\%, con respecto al año 2019, tramitándose 1.379 expedientes que afectaron a las relaciones laborales de 1.550 empresas y 4.880 trabajadores.

El 100\% de los expedientes tramitados se refieren a conciliación mediante procesos de mediación, siendo el 98,25\% de ellos de carácter individual y el 1,75\% de carácter colectivo.

Del total de expedientes tramitados, el 69\% han sido tramitaciones efectivas, estando presente empresas y trabajadores, y dando como resultado de "acuerdo 2 el 47,2\% de ellas, respecto al $52,8 \%$, en las que el resultado fue de "sin avenencia".

Tabla 11. TLR - Causas de los conflictos individuales durante el año 2020 en el TLR.

\begin{tabular}{|c|c|}
\hline & $\begin{array}{c}\text { CAUSAS DE LOS CONFLICTOS } \\
\text { INDIVIDUALES DURANTE EL AÑO 2020 EN } \\
\text { EL TLR (EN PORCENTAJE) } \\
\end{array}$ \\
\hline Despidos & $35,90 \%$ \\
\hline Cantidades & $33,50 \%$ \\
\hline Reconocimientos de derechos & $13,49 \%$ \\
\hline Despidos objetivos & $6,74 \%$ \\
\hline Infracciones y sanciones & $5,80 \%$ \\
\hline Extinción del contrato & $1,52 \%$ \\
\hline $\begin{array}{l}\text { Modificación sustancial de las } \\
\text { condiciones de trabajo }\end{array}$ & $1,02 \%$ \\
\hline Clasificación profesional & $1,02 \%$ \\
\hline
\end{tabular}

20 En este caso la memoria es de acceso libre y puede consultarse en la siguiente dirección: https://www.tlrioja.com/app/download/5816439112/BALANCE+TRIBUNAL+2020.pdf. Además, puede consultarse otros documentos recientes, como la memoria del año 2019, el informe de cuentas del año 2019 y el Plan de actuación de 2020. 
Tabla 12. TLR - Sectores afectados por los expedientes tramitados en el TRL en el año 2020.

\begin{tabular}{|l|c|}
\cline { 2 - 2 } \multicolumn{1}{c|}{} & $\begin{array}{c}\text { SECTORES AFECTADOS POR LOS } \\
\text { EXPEDIENTES }\end{array}$ \\
\hline Servicios & $49,30 \%$ \\
\hline Industrial & $31,76 \%$ \\
\hline Construcción & $7,40 \%$ \\
\hline Comercio & $8,60 \%$ \\
\hline
\end{tabular}

A fin de observar la tendencia de evolución y en base a los datos que refleja el Plan de Actuación del TLR en el año 2020 (Tribunal Laboral de La Rioja, 2020), entre los años 2015 y 2019, la evolución de expedientes tramitados, año a año, diferenciados entre conflictos individuales o colectivos ha sido la siguiente:

Tabla 13. TLR - Evolución anual de Conciliaciones en conflictos individuales y colectivos.

\begin{tabular}{|l|r|r|r|}
\cline { 2 - 4 } \multicolumn{1}{c|}{} & \multicolumn{1}{c|}{$\begin{array}{c}\mathrm{N}^{\circ} \text { Expedientes } \\
\text { Tramitados }\end{array}$} & \multicolumn{1}{c|}{$\begin{array}{c}\text { No Expedientes } \\
\text { Individuales }\end{array}$} & \multicolumn{2}{c|}{$\begin{array}{c}\mathrm{N}^{\circ} \text { Expedientes } \\
\text { Colectivos }\end{array}$} \\
\hline Año 2015 & 945 & 934 & 11 \\
\hline Año 2016 & 997 & 982 & 15 \\
\hline Año 2017 & 1.045 & 1.023 & 12 \\
\hline Año 2018 & 1.215 & 1.203 & 13 \\
\hline Año 2019 & 1.329 & 1.316 & 25 \\
\hline Año 2020 & 1.379 & 1.354 & \\
\hline
\end{tabular}

Puede observarse, a raíz de los datos contenidos en la tabla n ${ }^{\circ} 13$, que la evolución ha sufrido un aumento constante en términos globales en los últimos cinco años, pasando de 945 expedientes en 2015 a 1.379 en el año 2020.

Por último, el número de usuarios de la actividad del TLR (diferenciando entre número de empresas y número de trabajadores afectados), la evolución en los últimos cinco años ha sido la siguiente:

Tabla 14. TLR - Conciliaciones en conflictos colectivos.

\begin{tabular}{|l|r|r|}
\cline { 2 - 3 } \multicolumn{1}{c|}{} & \multicolumn{1}{c|}{$\begin{array}{c}\text { Empresas } \\
\text { afectadas }\end{array}$} & $\begin{array}{c}\text { Trabajadores } \\
\text { afectados }\end{array}$ \\
\hline Año 2015 & 1.307 & 2.835 \\
\hline Año 2016 & 1.105 & 2.295 \\
\hline Año 2017 & 1.324 & 2.901 \\
\hline Año 2018 & 1.402 & 2.402 \\
\hline Año 2019 & 1.495 & 2.426 \\
\hline Año 2020 & 1.550 & 4.880 \\
\hline
\end{tabular}




\section{V. Índice de recursos utilizados}

Fundación SIMA. (29 de septiembre de 2015). Normas de Funcionamiento y Procedimiento. Obtenido de https://fsima.bouge.es/wp-content/uploads/NORMAS-FUNCIONAMIENTOA4.pdf

Fundación SIMA. (2017). Estatutos de la Fundación "Servicio Interconfederal de Mediación y Arbitraje SIMA-F.S.P.”. Obtenido de https://fsima.bouge.es/wp-content/uploads/Estatutos-SIMAFSP-2017.pdf

Fundación SIMA. (junio de 2019). Guía de las buenas prácticas en mediación. Obtenido de https://www.fsima.es/wp-content/uploads/Sima-Guia-de-las-Buenas-Practicas-en-MediacionVersion-pagweb-1.pdf

Tribunal Laboral de La Rioja. (2020). Memoria Anual. Obtenido de https://www.tlrioja.com/app/ download/5816439112/BALANCE+TRIBUNAL+2020.pdf

Tribunal Laboral de La Rioja. (2020). Plan de Actuación. Obtenido de https://www.tlrioja.com/app/ download/ 5813772381/PLAN+DE+ACTUACION+2020.pdf

Tribunal Laboral de La Rioja. (s.f.). Estatutos. Obtenido de https://www.tlrioja.com/organizaci $\% \mathrm{C} 3 \% \mathrm{~B} 3 \mathrm{n} /$ funciones-y-normativa/ 